\title{
¿Cómo Abordan los Derechos Sexuales y Reproductivos Femeninos las ONGDs Andaluzas? Centralidad, Enfoques y Prácticas Situadas
}

\section{How do Andalusian NGDOs Address Sexual and Female Reproductive Rights? Centrality, Approaches and Located Practices}

\author{
José Hernández-Ascanio \\ jhascanio@uco.es @ https://orcid.org/0000-0002-9525-5377 \\ Facultad de Ciencias del Trabajo, Universidad de Córdoba-Campus Centro. \\ C/Adarve, 30. 14001 Córdoba. \\ Carmen de la Mata-Agudo \\ carmen.mata.agudo@uco.es @ http://orcid.org/0000-0001-5096-3399 \\ Facultad de Ciencias de la Educación y Psicología, Universidad de Córdoba. \\ Avda. San Alberto Magno, s/n. 14071 Córdoba
}

\section{INFO ARTÍCULO}

Recibido: 05-03-2020

Revisado: 05-06-2020

Aceptado: 08-06-2020

\section{PALABRAS CLAVE}

Derechos sexuales y reproductivos

Perspectiva de género

Cooperación al desarrollo

Tercer sector

Análisis de políticas públicas

\begin{abstract}
RESUMEN
Actualmente el abordaje de los Derechos Sexuales y Reproductivos de las mujeres en el ámbito de la cooperación al desarrollo experimenta una doble tensión: por un lado, los agentes de cooperación, especialmente las Organizaciones no Gubernamentales para el Desarrollo (ONGD) han priorizado su atención sobre esta temática, en la medida en la que se han aumentado los esfuerzos por prevenir, reducir y erradicar los efectos de las desigualdades de género en los diferentes ámbitos. Por otro lado, existe un claro retroceso en el ejercicio y disfrute de este tipo de derechos a nivel mundial. Una posible hipótesis explicativa que explica este desajuste puede estar en la diferente forma de conceptualizar los Derechos Sexuales y Reproductivos, asunto que condiciona las prácticas situadas de las organizaciones de manera significativa, a su razón de ser y a su naturaleza organizativa. Es por ello, que el objetivo del presente trabajo es indagar en cómo los Derechos Sexuales y Reproductivos se configuran y condicionan la razón de ser, la naturaleza y la práctica situada de las ONGD.

Se presenta un estudio de caso, cuyo objeto de estudio fue el sistema de cooperación al desarrollo en Andalucía. Se recurrió a una combinación de metodologías cuanti- cualitativas, aunque en el presente trabajo se priorizan los resultados encontrados a través de técnicas estructurales y dialécticas por ser especialmente significativos. Este trabajo pone de manifiesto que las ONGD andaluzas manifiestan un marcado interés y una tarea proactiva sobre este tipo de derechos y, a la par, un déficit estructural de competencias para poder gestionarlos de forma eficaz.
\end{abstract}




\begin{abstract}
For this, a case study is carried out, the object of which was the development cooperation system in Andalusia. A combination of quantitative - qualitative methodologies was used, although in this work we are going to prioritize the results derived from the structural and dialectical techniques as they are especially significant. This work shows that Andalusian NGDOs move between a tension marked by a proactive interest in this type of rights and a structural deficit of competences to be able to manage them effectively.
\end{abstract}

\title{
1. INTRODUCCIÓN
}

El último cuarto de siglo ha sido testigo de un interés creciente por el fomento de la Igualdad de Género (entendida ésta como aquella que se centra en las relaciones entre los sujetos, que no siendo iguales deben de poseer los mismos derechos, responsabilidades, oportunidades y acceso a los recursos, y no subordinada a sus identidades de género). En consonancia las organizaciones dedicadas a la cooperación y al desarrollo internacional han focalizado su tarea también en este objetivo. Estas actuaciones han estado enmarcadas en las prolíficas Conferencias Mundiales de Mujeres de Naciones Unidas realizadas en los últimos 40 años y en las reflexiones que de ellas han emanado.

La Conferencia celebrada en Beijing (ONU, 1995) va a ser determinante en la medida en la que promueve incorporar la perspectiva de género como herramienta en el diseño, ejecución y evaluación de políticas públicas en cualquier ámbito de actuación, mediante la aprobación unánime de la Declaración y Plataforma de Acción de Beijing. Por su parte, la Conferencia Internacional "Población y Desarrollo", celebrada en El Cairo en 1994, es la primera en establecer consensos sobre qué se entiende por salud sexual y reproductiva, y por derechos reproductivos, aunque como se verá más adelante, dichos consensos evolucionan paralelos al avance de los Estudios de Género. El cambio de perspectiva que supuso la Conferencia de El Cairo ha hecho posible la creación de políticas que favorezcan la participación de diferentes agentes sociales, especialmente ONG, en lo que respecta a la sensibilización y creación de estrategias para denunciar situaciones de discriminación contra el género femenino, educación en equidad y consecución y defensa de los Derechos de las mujeres.

A largo de los años noventa, las organizaciones internacionales prestaron especial interés a esta temática y la incorporaron, de forma restringida en los denominados "Objetivos de Desarrollo del Milenio" (ODM). En esta agenda los Derechos Sexuales y Reproductivos quedaron reducidos a un objetivo sobre la salud materna (ODM 5). En los últimos años hemos podido ver como los ODM han sido sustituidos por un planteamiento significativamente distinto, siendo denominados como "Objetivos de Desarrollo Sostenible" (ODS), en los que ha cristalizado un creciente esfuerzo por considerar los Derechos Sexuales y Reproductivos vinculados a dos objetivos prioritarios: el Objetivo 3 (con un enfoque de salud) y Objetivo 5 (con un enfoque de igualdad de género) (Organización de Naciones Unidad Mujeres, 2016). A pesar del citado esfuerzo, hay que subrayar un déficit en el abordaje de este conjunto de derechos en el intento de incorporar aspectos de la salud sexual, reproductiva y de la sexualidad: la orientación sexual, la diversidad en la identidad de género, el acceso a una educación sexual integral o el control del propio cuerpo por parte de las mujeres (Heidari, 2015).

Asimismo, junto con esta realidad, se observa una pérdida de Derechos Sexuales y Reproductivos de las mujeres a nivel mundial y, sobre todo, en países con Bajo Índice de Desarrollo Humano. Esto se debe, por un lado, al descenso de intervenciones en este ámbito, a la priorización de otras políticas en las instituciones y a cuestiones culturales y morales. Por otro lado, por el establecimiento de metas y plazos para alcanzar los ODS que desvían la atención de la agenda de los Derechos Sexuales y Reproductivos (Hawkes, 2014; Khosla, Say y Temmerman, 2015).

A pesar de que es imposible negar la necesidad de mantener la transversalización de la perspectiva de género (como incorporación de principios y prácticas de igualdad entre mujeres y hombres en todos los niveles y áreas de cualquier ámbito), referido a la sexualidad y a la salud sexual y reproductiva del género femenino precisa de una especial atención ya que en los últimos años se observa un retroceso en el reconocimiento y preservación de estos derechos. 
Las herramientas de cooperación internacional se han vuelto determinantes para poder llevar a cabo un trabajo en el Derechos Sexuales y Reproductivos en los países con bajo Índice de Desarrollo Humano. Analizar cómo se hace uso de estas herramientas es una prioridad de primer orden en el contexto que se explica (Yamin, 2019). A su vez, se observa que existe un vacío en esta temática, no sólo de origen académico sino también de evaluación interna por parte del propio sistema de cooperación al desarrollo (Coordinadora de Organizaciones para el Desarrollo España, 2019; Marrero, O'Kelly, Solsona y Zumarán, 2008).

Este trabajo plantea, a través de un estudio de caso, indagar en la manera en que las ONGD conceptualizan los Derechos Sexuales y Reproductivos femeninos y en cómo se relaciona este hecho con su naturaleza organizativa y su esencia. Asimismo, se plantea como la elección de unos modelos de desarrollo en el abordaje de los Derechos Sexuales y Reproductivos determina sus prácticas situadas. Es por ello, que se plantea como objetivo: identificar y describir las diferentes categorías relativas a la razón de ser, naturaleza organizativa, modelo de desarrollo y práctica situada, y ponerlos en relación con los enfoques teóricos predominantes sobre este tipo de derechos.

Este trabajo articula diferentes bloques con el objeto de abordar los interrogantes anteriormente indicados. En primer lugar, se presentarán de forma sintética los principales enfoques a partir de los cuales se conceptualizan y operativizan los Derechos Sexuales y Reproductivos, y a su vez, la evolución en su posicionamiento en el ámbito específico de la cooperación internacional al desarrollo. Esta presentación permite entender los enfoques como elemento clave en la configuración de los Derechos Sexuales y Reproductivos en las ONGD andaluzas, ya que dichos enfoques son el reflejo de los elementos estructurantes de la organización (misión, visión y valores) y sirven como facilitadores y mediadores de la práctica concreta de esas organizaciones (a partir de ahora denominada "práctica situada").

A continuación, se procederá a una breve descripción del diseño metodológico del estudio, y a una justificación de la elección de este. En el bloque central de este trabajo se procederá a hacer un análisis de cómo los Derechos Sexuales y Reproductivos, discutidos a partir de los enfoques anteriormente presentados, configuran las 4 dimensiones analíticas propuestas por Cuesta Fernández y Calabuig Tormo (2010) para analizar la razón de ser, naturaleza y práctica situada de los ONGD. La opción por esta herramienta analítica viene determinada por ser una de las pocas existentes en la literatura especializada, así como una de las más influyentes. Por último, la sección final presentará las conclusiones del estudio y las líneas de investigación de interés para el futuro.

\section{ENFOQUES TEÓRICOS EN EL ABORDAGE DE LOS DERECHOS SEXUALES Y REPRODUCTIVOS FEMENINOS}

El Diccionario de Acción Humanitaria y Cooperación al Desarrollo promovido por el Instituto de Estudios sobre Desarrollo y Cooperación Internacional define el concepto Derechos Sexuales y Reproductivos como:

\footnotetext{
"Derechos de las mujeres y los hombres a tener control respecto de su sexualidad, a decidir libre y responsablemente sin verse sujetos a la coerción, la discriminación y la violencia; el derecho de todas las parejas e individuos a decidir de manera libre y responsable el número y espaciamiento de sus hijos y a disponer de la información, la educación y los medios para ello, así como a alcanzar el nivel más elevado de salud sexual y reproductiva" (Pérez de Armiño, 2000, p. 71).
}

Como se puede comprobar, en dicha definición existe una clara inspiración de los principios formulados en la Conferencia sobre Población y Desarrollo (ONU, 1994), y de la Cuarta Conferencia Mundial sobre la Mujer (ONU, 1995). A su vez, esta definición recoge aportaciones propuestas originalmente por el movimiento feminista (De Barbieri, 1999).

El hecho que se establezcan distinciones entre la categoría "mujer" y la categoría "persona" en el momento de construir a los sujetos de derecho, evidencia una tensión que ha aumentado en paralelo al aumento de las reivindicaciones en el ámbito de la sexualidad y de la reproducción, y la centralidad que estas han tomado en la agenda del movimiento feminista.

En la medida en la que avanzan las acciones y las reflexiones sobre los Derechos Sexuales y Reproductivos promovidos por organismos nacionales e internacionales, así como por los movimientos sociales vinculados 
a los últimos avances que ha procurado el movimiento feminista (centrados en el empoderamiento de la mujer, y en el control de su propio cuerpo, la contracepción y la erradicación de los diferentes tipos de violencia que generan respectivamente) se evidencia la dificultad para conceptualizar estos derechos de una forma consensuada e inequívoca (González Luengo y Gutiérrez Esteban, 2011).

Autorías como Jelin (1993), por ejemplo, plantean una posible tensión latente, entre las posiciones que defienden el derecho al control y autonomía sobre el propio cuerpo, y por otro demandan al sexo masculino que asuma de forma igualitaria las posibles consecuencias del ejercicio de su propia sexualidad. Esta plantea un proceso de negociación, a la vez que posibilita significativas preguntas en el sentido de si los derechos reproductivos son derechos exclusivos de las mujeres o, sin embargo, son derechos de carácter relacional. Incluso, plantea un interrogante más incisivo reflexionando sobre si es posible identificar un actor social con capacidad, autoridad y legitimidad para arbitrar los conflictos emergentes en este ámbito.

Para Shallat (1993), la principal causa que explica por qué los Derechos Sexuales y Reproductivos no han podido ser formulados de una manera adecuada en cuanto a precisión y claridad está vinculada cultural e históricamente a una dimensión del ser humano considerada como privada, y por lo tanto, se han mantenido al margen de las esferas política y legislativa. Igualmente, plantea argumentos que explican que estos derechos en su desarrollo pueden entrar en conflicto con la calidad en la prestación y acceso a la atención o el consentimiento informado. Los Derechos Sexuales y Reproductivos están íntimamente relacionados con la esfera de la sexualidad, en la medida en la que el ejercicio de la sexualidad suele verse condicionado por la posición subordinada del género femenino, y muchas veces por la definición de la identidad femenina asociada a la maternidad.

Para poder llevar a cabo un abordaje de los Derechos Sexuales y Reproductivos en el ámbito de la cooperación al desarrollo es necesario tener presente (ya que no hay disponible una definición de carácter canónico sobre qué son y que suponen esta categoría de derechos) cuáles son los diferentes enfoques desde los cuales es posible enfrentar el análisis, así como cuáles son las posibles implicaciones, que pueden ir desde la construcción de los discursos teóricos hasta los impactos concretos sobre los cuerpos, y las vidas del género femenino, derivadas de los mismos.

A continuación, procedemos a hacer una breve exposición de los enfoques de referencia en la materia de Derechos Sexuales y Reproductivos utilizando como referencia los trabajos de González Guerrero y Pajares Sánchez (2012).

El primero de los enfoques a tener en cuenta sería el que se ha venido a denominar como Enfoque de Derechos. Su principal característica es que pretende integrar los Derechos Sexuales y Reproductivos como elementos exigibles a partir de la jurisprudencia y acervo normativo multinivel (nacional e internacional) en materia de derechos humanos y otros derechos de las mujeres y del género femenino (Pavón Piscitello, 2012). Un ejemplo de estos marcos de referencia serían la Convención para la Eliminación de Todas las Formas de Discriminación contra la Mujer (CEDAW)(ONU, 1979), o la Convención Interamericana para Prevenir, Sancionar y Erradicar la Violencia contra la Mujer (Convención Belém do Pará) en América Latina (OEA, 1994).

Autorías como De Barbieri (1999) ponen de manifiesto que el déficit y la ambigüedad en la formulación de los Derechos Sexuales y Reproductivos llevada a cabo por las diferentes Conferencias Mundiales auspiciadas por la ONU, se pueden explicar principalmente por el hecho de que estos derechos se encuentran en un estatus jurídico considerado como "débil". Se han consensuado en espacios que no tienen capacidad vinculante para los participantes, porque no existe una asignación clara de atribuciones a los diferentes agentes implicados (Estado, sociedad civil y organizaciones del género femenino). Y en último lugar, porque no existen mecanismos sancionadores con carácter internacional que respalden alguno de estos derechos.

El siguiente enfoque a tener en cuenta es denominado como Enfoque en Salud. Concretamente, son tres, los puntos focales que han marcado la evolución de este enfoque (Ballester Añon, 2015). En primer lugar, situar la salud de las mujeres (y los Derechos Sexuales y Reproductivos contenidos en ésta) desde una construcción welfarista del bienestar. El género femenino es construido como sujeto pasivo dentro de las intervenciones de desarrollo, asumiendo que su función principal es participar del mismo desde el rol de "madres y cuidadoras del bienestar familiar" (Ballester Añon, 2015). Este es un modelo de claro corte asistencialista. Las intervenciones más frecuentes en el mismo tienen como objetivo principal dotar al género femenino de las 
competencias básicas en el desempeño del rol de procreación y cuidado, pero no se cuestiona el acceso a los derechos ni la compensación de las posibles desigualdades en salud de una posible perspectiva de género. Este se desgaja en el denominado Enfoque de salud materno infantil (González Guerrero y Pajares Sánchez, 2012) el más tradicional y utilizado a nivel global, y que se constituye como perspectiva hegemónica en la Declaración del Milenio (ONU, 2000),y posteriormente en los ODS (Organización de Naciones Unidas, 2015). Ha sido una de las perspectivas con mayores críticas por parte del movimiento feminista y de las organizaciones de mujeres, ya que supone un reduccionismo sobre la potencialidad a los Derechos Sexuales y Reproductivos a la dimensión específica del rol reproductivo y de crianza. Y sitúa a las mujeres en posiciones muy pasivas con respecto a la implementación de los diferentes proyectos y programas (González Guerrero y Pajares Sánchez, 2012).

Un segundo punto focal dentro del Enfoque en Salud se da a partir de la década de los años 60 y 70 . En este momento, se acentúa por un lado, el carácter reproductivo del género femenino (en la media en la que se la construye como principal agente que garantiza la supervivencia de los menores) y por otro, se promociona su figura como "Agente de salud" ya que es reconocido como el principal proveedor de servicios y recursos que condicionan aspectos fundamentales de la salud y el bienestar social de la comunidad en su conjunto (Ballester Añon, 2015).

El tercer punto focal aparece en los años 90, y plantea una reorientación en la que la salud reproductiva del género femenino se plantearía como un derecho humano independiente no condicionado a la crianza o a la prestación de cuidados.

Este punto focal se concretó en una variante del Enfoque en Salud denominado Enfoque Integral de la Salud (González Guerrero y Pajares Sánchez, 2012) y a partir del mismo se explican un número significativo de programas de Educación Sexual y de atención sanitaria abordan la sexualidad desde una dinámica de prevención y asistencia a procesos de enfermedad. El enfoque de salud integral aborda tanto la salud reproductiva como la salud sexual, entendida esta como la expresión de un estado de bienestar integral (físico, emocional, mental y social) en el ámbito de la sexualidad, que además es de carácter complejo y aparece determinado por múltiples factores (biológicos, sociales, de medio ambiente, de acceso a servicios sociosanitarios, etc.) en el que las relaciones de género van a ser especialmente significativas. En este espacio debe ser incluida la autonomía sexual para expresar y manifestar los deseos, el placer, las prácticas sexuales, así como orientación e identidad sexual. La Conferencia de El Cairo aplica este enfoque integral cuando habla de salud reproductiva.

Un tercer enfoque es el denominado Enfoque de Laicismo y Ciudadanía, que implica dos cuestiones para tener en cuenta. Tiene como punto de partida la construcción y reivindicación de la mujer como sujeto político, plenamente activo y capaz de decidir sobre su cuerpo, su sexualidad y su deseo. Además, este enfoque asume que los Derechos Sexuales y Reproductivos deben ser construidos como asuntos de dimensión pública (frente a aquellos enfoques que tienden a recluirlos al espacio privado-doméstico). Por tanto, deben ser reconocidos y defendidos por y para el conjunto de la ciudadanía, dentro de una organización política del estado con orientación laica y democrática. Una última atribución a este enfoque es su carácter instrumental frente a otros más sustantivos, lo que le otorga la posibilidad de evitar o superar posiciones utilitaristas o dicotómicas (cuya principal característica es que los conflictos entre derechos son utilizados para menoscabar los derechos y prácticas de ciudadanía de las mujeres) (Pavón Piscitello, 2012).

Un cuarto enfoque es el Enfoque de Autonomía y Empoderamiento. Sus principales características son, en primer lugar, que se articula en torno al derecho a decidir, lo que lo dota de robustez y lo anuda con el Enfoque de Ciudadanía, tanto en lo que tiene que ver con las múltiples reivindicaciones de incidencia política como, en el trabajo de base de los movimientos en torno a los cuales se organizan las mujeres. En segundo lugar, considerar el empoderamiento como un proceso que se implementa en paralelo a los propios procesos de desarrollo, enriqueciendo a estos mediante dinámicas de reflexión y cuestionamiento crítico.

La práctica situada desde este enfoque implica necesariamente que el género femenino es el protagonista del proceso, liderando los cambios y con capacidad plena de movilización y acción con respecto a sus propios derechos y necesidades. Como consecuencia de la aplicación de este enfoque, se evita la aplicación de proyectos y programas de carácter asistencialista. 
Su conexión con el Enfoque de Ciudadanía converge en la explicación de los procesos de empoderamiento como procesos individuales con base en la autonomía personal pero que sólo encuentran sustento en la colectividad, a partir de la posibilidad de garantizar el estatuto de ciudadanía. Algo que Marcela Lagarde y de los Rios (2005) define como el "tenerse a una misma como la protagonista de la vida, vivirse como el propio sentido de la vida".

El último enfoque que es necesario considerar es el Enfoque Intercultural, cuya principal característica es que asume la diversidad de construcciones socioculturales en torno al ámbito de la sexualidad y la reproducción. Esta asunción puede plantear un debate entre los diferentes derechos culturales, especialmente en relación con este ámbito, y en otros de los derechos humanos, especialmente cuando los sujetos que los ejercen son las mujeres. Este enfoque identifica discrepancias complejas en el entendimiento de los derechos humanos y derechos culturales, y ha supuesto el escenario en el que se han desarrollado profundas discusiones, que han puesto de manifiesto que en la mayoría de las prácticas culturales los derechos de las mujeres se han instrumentalizado y se han visto condicionadas a hacer efectivo un control sobre el cuerpo de estas (González Guerrero y Pajares Sánchez, 2012). La autora feminista Seyla Benhabib considera que preguntar acerca de la concordancia entre los derechos humanos y las cosmovisiones supone una discusión polarizada que se asemeja a la dicotomía "indiferencia e intervencionismo" (Benhabib, 2010). Esta autora propone que es imprescindible clarificar si las minorías vulnerables tienen fundamento para reclamar derechos culturales, y en el caso de ser así, identificar cuáles son (Benhabib, 2010).

Utilizando como punto de partida este cuestionamiento, el Enfoque intercultural de los Derechos Sexuales y Reproductivos defiende que resulta necesario reconocer la diversidad interna a los propios sistemas culturales (lo que permitiría anular una tendencia homogeneizadora). Igualmente intentar neutralizar las dinámicas asimilacionistas, recurriendo para ello a la apertura de espacios equitativos de diálogo intercultural que den oportunidad a las minorías culturales para definir, articular y ejercer en condiciones de igualdad sus Derechos Sexuales y Reproductivos, y dentro de ellas, especialmente a las mujeres.

\section{METODOLOGÍA}

El estudio original se abordó como una investigación empírica de carácter cuanti-cualitativo y participativa, utilizando como informantes a organizaciones que desempeñan su actividad en el marco de la cooperación andaluza (a través de los agentes sociales, técnicos y responsables e informantes privilegiados). En este plano, las técnicas de investigación que se desarrollaron fueron las siguientes.

Aplicación de una encuesta dirigida a responsables de las organizaciones inscritas en el Registro de Agentes de la Cooperación Internacional para el Desarrollo en Andalucía (RACDA) a fecha de diciembre de 2018. El cuestionario se aplicó al total de organizaciones (no se hizo selección muestral al considerar que era un universo de estudio abordable) y se proporcionó un cuestionario on -line auto-completable. Sobre las 347 organizaciones contactadas, se obtuvieron un total de 207 registros lo que garantiza la representatividad estadística con 50\% de heterogeneidad, un margen de error de 5 y un intervalo de confianza del 95\%.

Se recurrió a la realización de 23 entrevistas para completar la información recibida. Se utilizó la entrevista semiestructurada, que se realizó individualmente, a técnicos y responsables de Organizaciones adscritas a la Coordinadora Andaluza de Desarrollo. La selección de informantes se hizo de forma intencional siguiendo estrategia de "bola de nieve" hasta obtener una saturación del discurso. En relación al uso de estas técnicas fueron tenidas en cuenta las recomendaciones del "The Standards for reporting qualitative research" (O'Brien, Harris, Beckman, Reed y Cook, 2014). Los datos se han triangulado a través de la realización de dos talleres colaborativos en los que participaron 16 técnicos pertenecientes a 12 organizaciones de la citada Coordinadora, que previamente habían participado en las entrevistas individuales.

Las técnicas de recogida de datos abordaron 4 bloques de información: variables de descripción y caracterización de la ONGD; referentes ideológicos y teóricos a partir de los cuales se conceptualizaban las Derechos Sexuales y Reproductivos; importancia reconocida a este tipo de derechos dentro las prácticas de las ONGD y análisis de prácticas concretas en este ámbito. 
El análisis de los datos se llevó a cabo aplicando un enfoque de Teoría Fundamentada (Glaser y Strauss, 1967) sobre los elementos de contenido y contextuales obtenidos. Esto permitió establecer una serie de categorías que explican cómo los diferentes enfoques utilizados para trabajar los Derechos Sexuales y Reproductivos desde las ONGD andaluzas configuran su razón de ser, su naturaleza y su práctica situada, así como permitió identificar posibles condicionantes en dicha configuración.

El espacio temporal al que hace referencia el estudio abarca el período 2012 -2016, por ser a partir de esta fecha el momento en el que se encuentran vigentes los denominados Objetivos de Desarrollo Sostenible que van a suponer un cambio radical en la práctica de la cooperación internacional al desarrollo.

Los resultados obtenidos a partir de técnicas distributivas permiten una mejor comprensión de aspectos tales como el papel que ocupan los derechos Sexuales y reproductivos femeninos en la razón de ser y naturaleza de las ONGD andaluzas. Asimismo, los datos derivados de técnicas estructurales y dialécticas aportarán datos relevantes sobre la relación de este tipo de derechos con los modelos de desarrollo aplicados por las ONGD, así como su práctica situada.

\section{RESULTADOS Y DISCUSIÓN}

\subsection{Orientación y ámbito de actuación de las ONGS andaluzas con respecto a los derechos sexuales y reproductivos}

Según la Ley 23/1998, de 7 de julio, de Cooperación Internacional para el Desarrollo, se consideran organizaciones no gubernamentales de desarrollo (ONGD):

"aquellas entidades de Derecho privado, legalmente constituidas y sin fines de lucro, que tengan entre sus fines o como objeto expreso, según sus propios Estatutos, la realización de actividades relacionadas con los principios y objetivos de la cooperación internacional para el desarrollo".

Gómez Galán y Sanahuja (1999, p. 224) argumentan que las ONGD son un conjunto de organizaciones en las que predomina la diversidad en relación a sus "principios rectores, prácticas, métodos de trabajo, modalidades y sectores de actuación, el volumen, las fuentes de financiación y los vínculos institucionales". Esta diversidad es una de las principales potencialidades de este tipo de organizaciones, pero también uno de los principales impedimentos a la hora de poder llevar a cabo una definición o una clasificación de las mismas.

Una forma simplificada y asequible de hacerlo sería utilizar la orientación y el ámbito preferencial de actuación en el que se aplican. Se toman como referencia: los reconocidos por los organismos internacionales (Gómez Galán y Sanahuja, 1999): realización de proyectos de desarrollo, acciones en materia de ayuda por emergencia, actividades de sensibilización y concienciación social, educación para el desarrollo e incidencia pública - política, elaboración de estudios o acciones en el marco del ámbito del "comercio justo". Para el caso de Andalucía, aunque algunas de las organizaciones tienden a especializarse en alguno de estos ámbitos de aplicación (especialmente el que tiene que ver con la educación para el desarrollo) existe una tendencia mayoritaria de actuar simultáneamente en diferentes líneas con la finalidad de aprovechar las sinergias que surgen de actuar de una forma integrada, además de mejorar su posicionamiento de oportunidad frente a la captación de recursos.

En la figura 1 se puede observar cómo definen las ONGD inscritas en el RACDA en referencia al área en el que desarrollaban su actividad principal.

\subsection{Los derechos sexuales y reproductivos femeninos en la estructura y modelo organizativo de la ONGD andaluzas}

Una cuestión fundamental a tener en cuenta va a ser la posición de los Derechos Sexuales y Reproductivos en las disposiciones y modelos organizativos de la entidad. Por un lado, reflejan las diferentes construcciones 


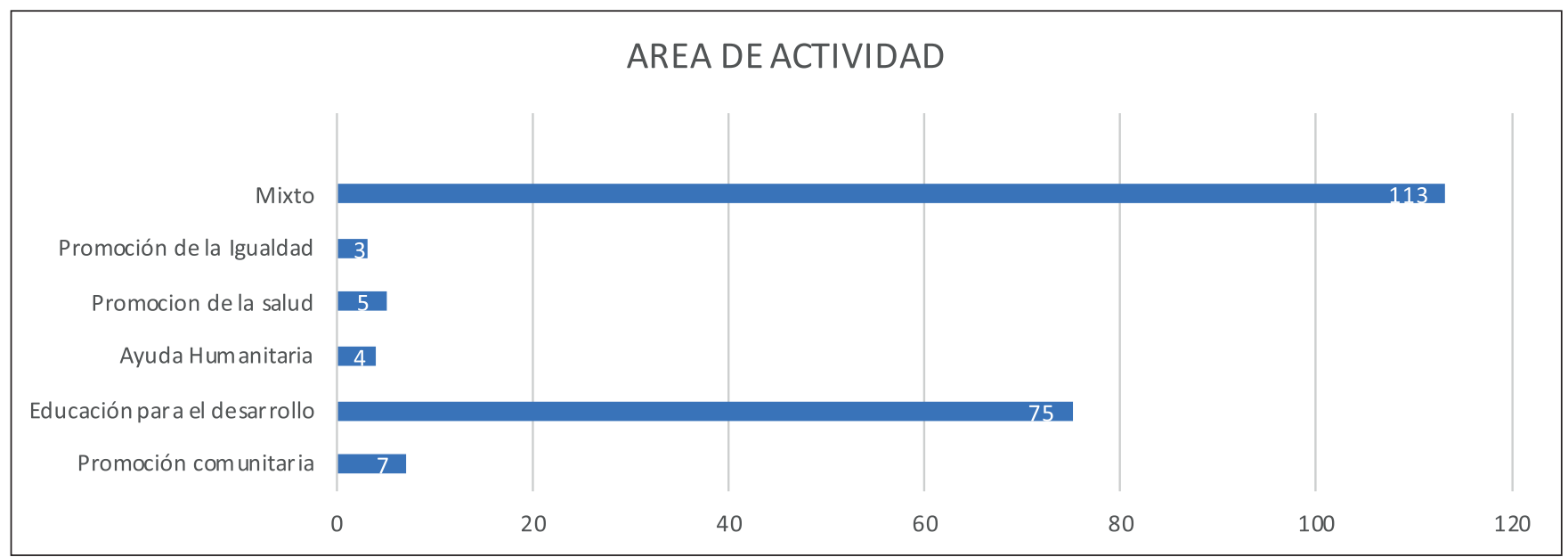

Figura 1. Área de actividad de las ONGD del RACDA. Fuente: Hernández - Ascanio (2020).

ideológicas de la organización sobre esa cuestión en concreto y por otro, orientan la práctica situada de sus acciones (Cuesta Fernández y Calabuig Tormo, 2010).

Preguntadas las organizaciones con respecto a cuál es el posicionamiento político, es decir, cómo se encaja dentro de su "Identidad, visión y valores" la defensa de este tipo de derechos se observan aspectos interesantes. En primer lugar, la mayoría de estas entidades no los consideran explícitamente. En consecuencia, estos no son contemplados de forma programática en los planteamientos estratégicos. Esto puede ser en parte explicado por el bajo nivel de especialización de las organizaciones encuestadas (Marrero et al., 2008; Coordinadora de Organizaciones para el Desarrollo España, 2019). Las que sí los tienen explicitados, utilizan como referencia básicamente los Enfoques de Derechos y el Enfoque de Empoderamiento. Teniendo en cuenta los resultados que muestran el valor de los alineamientos estratégicos en la aplicación y resultados, y que manifestaba la priorización en el ámbito de la salud y de la promoción de género se desprende que los posicionamientos políticos que se relacionan con los Derechos Sexuales y Reproductivos se acaban materializando en intervenciones aplicadas en el ámbito de la salud y de la promoción del género, entendidos estos ámbitos en sentido amplio. Sólo en organizaciones altamente especializadas se encuentra un alineamiento entre los posicionamientos políticos en esta materia y la operativización que se aplica a los mismos mediante planificaciones estratégicas e intervenciones concretas (Coordinadora de Organizaciones para el Desarrollo España, 2019).

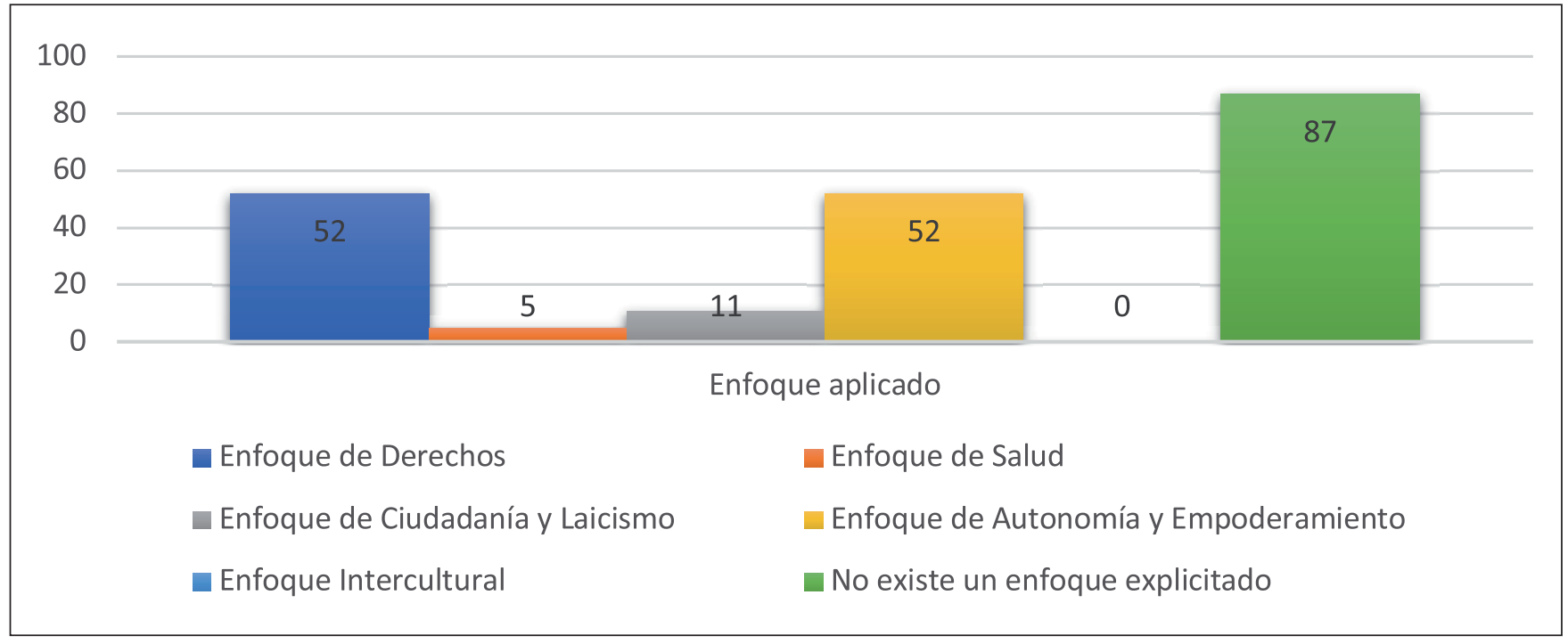

Figura 2. Enfoque político aplicado por la organización en la consideración de los Derechos Sexuales y Reproductivos. Fuente: Hernández - Ascanio (2020). 
En este contexto no es difícil entender que, cuando las organizaciones orientadas a la cooperación al desarrollo sitúan la intervención en materia de Derechos Sexuales y Reproductivos en sus alineamientos estratégicos, lo hacen a partir de dos grandes focos: el ámbito del género (entendido esto como las diferentes practicas orientadas a reducir las múltiples desigualdades derivadas de la construcción social sobre la forma de "ser hombre" y "ser mujer" en un momento histórico y en un territorio concreto (Arango Gaviria y Viveros Vigoya, 2011)) y el ámbito de la salud. Junto con esto, también resulta significativo que casi el 40\% de las organizaciones encuestadas no sitúan esta temática de manera específica en ninguno de los ejes de su planificación estratégica, lo que va muy en consonancia con lo argumentado con respecto a la invisibilización y secundarización de este tipo de derechos frente a otros a la hora de definir intervenciones (Olmo Gascón, 2018).

$\square$ SALUD $\square$ GENERO $\square$ EDUCACION $\square$ DEMOCRACIA Y SOCIEDAD $\square$ GOBERNANZA $\square$ OTRO $\square$ NINGUNO

\begin{tabular}{|c|c|c|c|c|}
\hline EJE ESTRATÉGICO & 52 & 52 & 130 & 86 \\
\hline
\end{tabular}

Figura 3. Consideración de los Derechos Sexuales y Reproductivos en la planificación estratégica de la organización. Fuente: Hernández - Ascanio (2020).

\subsection{Los derechos sexuales y reproductivos femeninos en los modelos de desarrollo de las ONGD andaluzas}

La consideración sustantiva de las temáticas relacionadas con los enfoques de género o la consideración del género femenino y sus derechos como sujetos prioritarios va a tener una representación escasa en las organizaciones estudiadas, lo que puede suponer un indicador de la secundarización o disolución de este tipo de derechos en cuanto a las prioridades de acción de la organización.

Esta idea se ve confirmada por los informantes:

"Estos derechos no son trabajados de manera concreta en nuestra organización ni en muchas de las organizaciones con las que solemos trabajar. No suelen estar dentro de nuestros debates estratégicos porque no están realmente especificados. Pensamos que forman parte de un conjunto más amplio de derechos de los que no se pueden desvincular" D7

Lo que sirve como argumento para profundizar en las categorías a partir de las cuales las organizaciones conceptualizan los Derechos Sexuales y Reproductivos femeninos.

Un primer análisis, nos permite identificar cómo aparecen de forma interconectada tres categorías que operativizan el concepto que las organizaciones estudiadas utilizan sobre los Derechos Sexuales y Reproductivos.

La primera categoría, en la que se identifica un mayor nivel de coincidencia entre los informantes, es conceptualizar este tipo de derechos como "El derecho a ejercer control sobre el propio cuerpo y sobre la reproducción de forma planificada".

"De igual manera consideramos los derechos reproductivos al margen de la sexualidad, como una opción personal de las mujeres, eligiendo en qué momento y circunstancias quieren o no asumir su maternidad y ser atendidas en un 
sistema sanitario público, de calidad y gratuito, tanto en lo que se refiere en los procesos de embarazo, parto y puerperio o en la circunstancia de querer interrumpir embarazos no deseados" D6

"Derechos sexuales: es el derecho a la libre expresión de la sexualidad de cada persona y a su vivencia, desde el respeto individual y social a todas las expresiones. Derechos reproductivos: es la libertad de decisión de las mujeres de cuando, como y con quien reproducirse, lo que conlleva el acceso a métodos anticonceptivos sin que importe nivel socio económico ni ninguna otra circunstancia". D7

"Los relativos a la reproducción biológica de la vida (aborto, no eliminación de fetos por razones de género, etc.)" D11

"Derecho a vivir una sexualidad y una vida sexual plena, segura y satisfactoria. Tener acceso a información veraz y adaptada a las necesidades de cada persona, contexto y realidad, así como a servicios referidos a salud sexual, que permitan a cada persona tomar las decisiones que le permitan una vivencia plena de su vida sexual. Decidir libremente y sin coacciones si ser, o no ser, madre o padre, cuándo, cómo y con quién. Que las personas sean respetadas en las decisiones que adoptan en relación con sus propios cuerpos" D12

"Que cada persona tenga forma libre el control sobre su sexualidad, cuerpo para poder decidir sobre cualquier aspecto referido a los mismos" D26

"Los Derechos Sexuales y Reproductivos son derechos humanos fundamentales relativos al libre ejercicio de la sexualidad sin riesgos, al placer físico y emocional, a la libre orientación sexual, a la libre elección del número de hijos e hijas, a la protección de la maternidad, encaminados al logro de una mejor calidad de vida" D27

Consecuentemente, esta categoría está totalmente alineada con el Enfoque en Salud que ya se ha expuesto brevemente en anteriores epígrafes. Este enfoque es predominante en las organizaciones cuyo sector prioritario de actuación es la intervención en salud, las que se desempeñan en el ámbito de la educación para el desarrollo, y en un número significativo de organizaciones que tienen una aplicación multisectorial.

Una explicación acerca de la hegemonía de este enfoque puede estar relacionado con ser el más clásico, y ampliamente asentado en la práctica habitual de los agentes de cooperación. Este hecho evidencia el triunfo del modelo biomédico como paradigma dominante en la construcción de la sociedad moderna occidental (Lozano, 1999; González Guerrero y Pajares Sánchez, 2012). Sin embargo, también debe considerarse que este es un enfoque que se ajusta a los intereses de las agencias financiadoras de la práctica de cooperación y que por lo tanto, este posicionamiento puede ser una decisión estratégica de las ONGD andaluzas para situarse adecuadamente en la captación de fondos, cada vez más limitados (Marrero et al., 2008).

La segunda categoría emergente que aparece con mayor consistencia es construir estos derechos como "El derecho a decidir sobre la propia vida en términos de autonomía, desarrollo personal y autorrealización". Esta visión se presenta ubicada entre las posturas tradicionales y aquellas posiciones más afines a un Enfoque de Empoderamiento. Esta es la categoría que aparece como predominante entre las organizaciones especializadas en el ámbito del género aunque también es asumida de manera significativa por organizaciones que trabajan en el ámbito de la educación para el desarrollo o que desarrollan actuaciones en múltiples sectores de actividad (González Guerrero y Pajares Sánchez, 2012).

"Los derechos que tienen las mujeres a decidir sobre si mismas" D1

"Son los derechos que velan por la libertad de decidir personal y libremente sobre la sexualidad que cada uno quiera, así como a nivel reproductivo" D3

"Derecho sexual y derecho reproductivo son la libertad de decidir cómo sobre la propia vida sexual y reproductiva. Decidir sobre el derecho a casarse, a tener hijos, a tener pareja sexual, etc." D9

"Derechos de la persona al pleno desarrollo de su sexualidad, por una parte, y a la realización personal a través de su capacidad reproductiva o la posibilidad de planificarla de acuerdo con sus intereses vitales, por otra" D21

El eje transversal de actuación de estas organizaciones es la educación, lo que puede proveerlas de una mayor capacidad reflexiva-autocrítica con respecto a sus propias acciones, de tal forma que les resulta más hábil pensar el desarrollo en sentido procesual (y no sustantivo como en otros enfoques) e identificar los Derechos Sexuales y Reproductivos en sentido mucho más abierto y acorde a esos principios procesuales (Olmo Gascón, 2018).

La tercera categoría identificada es de carácter mucho más minoritario entre las organizaciones. Aparece relacionada con organizaciones que no tienen un alto nivel de especialización en cuanto al ámbito de actuación. Con respecto a los resultados arrojados en otros enfoques, su formulación es poco precisa, pero supone 
una elaboración alternativa al Enfoque de Derechos en términos de los Derechos Sexuales y Reproductivos como "El derecho a la identidad"

"Son aquellos que hacen referencia al respeto y defensa de la identidad y disfrute sexual de cualquier persona, y con derechos reproductivos" D2

"Derecho de todas las personas a elegir libremente su sexualidad, sin ser discriminado por esta razón" D4

"Está dentro de los Derechos Humano, respeto a la diversidad sex., contra la discriminación por orientación sexual, contra la privación de libertad sexual, por la autonomía e integridad sexual., por el derecho a la identidad sexual..." D22

Este último enfoque ha ido adquiriendo con el tiempo una mayor presencia en la medida en la que las acciones de cooperación promovidas por las administraciones públicas españolas han asumido el Enfoque Basado en Derechos como uno de sus principales elementos sustentadores (Prado Lallande, 2010).

Como se puede constatar, las organizaciones manifiestan una gran dificultad a la hora de conceptualizar los Derechos Sexuales y Reproductivos, y eso se hace mucho más evidente a la hora de considerar si son derechos autónomos o si, por el contrario, se tratan de derechos vinculados. A este respecto es significativo comprobar que sólo un tercio de las organizaciones encuestadas consideran que estos derechos no son derechos autónomos entre sí. Esto lo explican a partir de los siguientes argumentos:

"Normalmente suelen ir asociados, ya que normalmente si se viola el derecho sexual también puede verse violado en el derecho reproductivo. Pero se pueden dar casos en los que son autónomos, como en casos de homosexuales, control de natalidad de gobiernos, casos monoparentales, etc. Por lo que están relacionados, pero son autónomos" D5

"Personalmente entiendo que el ejercicio de los derechos sexuales, entendido en un sentido amplio, implica el ejercicio de los derechos reproductivos" D11

"Se complementan en gran medida, pero protegen libertades y garantías diferenciadas" D16

"Porque la libertad de elección es tanto para el cuerpo como para el ejercicio de la sexualidad" D23

En esta argumentación, la relación entre Derechos Sexuales y Reproductivos se realiza a partir de que los derechos reproductivos suponen una particularidad de los derechos sexuales y siempre desde la aplicación de una lógica corpórea. Dicho de otra manera, los derechos reproductivos son una expresión de los derechos sexuales y ambos están condicionados por el derecho de las mujeres al "uso y disfrute" de su propio cuerpo. En cierta forma, cuando aquí expresan esta relación se están expresando los Derechos Sexuales y Reproductivos como un derecho al cuerpo, lo que coincide plenamente con la definición más consensuada que llevan a cabo con respecto de estos derechos (Heidari, 2015).

Sin embargo, también podemos encontrar organizaciones que desvinculan unos y otros derechos. La particularidad en este caso estaría en que se presta una mayor autonomía e independencia al ámbito de la sexualidad y se vincula a esta con elementos de carácter identitario y de autorrealización. Esta es una postura en la que se identifican afinidades en los Enfoques de Derechos, y más marginalmente, al Enfoque de Empoderamiento (Pavón Piscitello, 2012).

"Considerar que los derechos sexuales están ligados a los reproductivos, es reducir un derecho a la sexualidad y a su disfrute" D3

"La sexualidad no tiene por qué ir asociada a la reproducción, siendo un concepto muchos más amplio" D4

"Considero que son derechos autónomos. La sexualidad es más compleja, tiene más funciones y abarca muchos más aspectos que la reproducción. No debemos limitar la sexualidad a la reproducción" D19

"El derecho a decidir cada persona como vive su sexualidad y decidir sobre su cuerpo conlleva al reconocimiento de que cada cual puede y debe decidir sobre servicios de salud como la contracepción, matrimonio o no, modelos familiares, hijos sí o no... en el contexto de una sexualidad vivida libre de violencia" D20

\subsection{Los derechos sexuales y reproductivos en la práctica situada de las ONGD andaluzas}

En lo que se refiere a la realización de acciones directamente relacionadas con la temática de los Derechos Sexuales y Reproductivos. se observa que un elevado número de organizaciones han implementado actividades. 
Los datos reflejan que más del $60 \%$ de las organizaciones estudiadas implementaron al menos una 1 acción, y que fue casi el $40 \%$ el que había ejecutado 3 o más proyectos para el periodo estudiado.

Si se presta atención al origen de los recursos con los que se sufragan estas actividades, se observa que la dependencia de recursos públicos para la ejecución de estos proyectos es limitada, ya que sólo la mitad de los proyectos ejecutados se nutría de fondos públicos para su realización, y que el monto de dichas ayudas oscilaba entre el 15 y el $75 \%$ sobre el total de los costes de los proyectos. La escasa presencia de fondos públicos para financiar este tipo de intervenciones es explicada por las propias organizaciones en términos de que los proyectos presentados no coincidían con las orientaciones ideológicas de los gestores públicos del momento (el 83\% de las organizaciones contactadas manifestaban estar "bastante de acuerdo" o "totalmente de acuerdo" con esta afirmación) y por el hecho de que no identificaban en las entidades financiadoras personal técnico cualificado de manera suficiente con este tipo de derechos, lo que se traducía en la penalización de proyectos que no se alineaban con los enfoques hegemónicos en el sistema (Coordinadora de Organizaciones para el Desarrollo España, 2019).

En la mayoría de las ocasiones, los proyectos en este ámbito son financiados a partir de recursos propios, lo que de una manera puede servir como indicador del interés que manifiestan las organizaciones sobre estas temáticas (aunque no sea de una forma sistemática y coherente con su definición organizacional) a la misma vez que posibilita la aplicación de enfoques más innovadores. Un aspecto negativo derivado de esta situación sería que la implementación de proyectos en esta temática estaría más condicionada por la disponibilidad o no de fondos.

Al realizar un análisis del contenido de estas actuaciones, se puede observar que las mismas se concentran en torno a dos grandes áreas de interés. Por un lado, acciones en el ámbito de la educación, sensibilización y empoderamiento tanto de colectivos específicos como de la ciudadanía en general. Por otro lado, se hallan acciones de intervención específicamente diseñadas para poblaciones dianas mucho más concretas y diferenciadas. Pese a esa aparente polarización temática, la misma es de carácter bastante ficticio ya que las organizaciones manifiestan que con frecuencia se enfrentan al problema de distinguir el límite claro entre un tipo y otro de acción, al entender que existe una fuerte interseccionalidad entre los diferentes aspectos relacionados con los derechos Sexuales y Reproductivos que exige de un abordaje multifocal (Heidari, 2015).

"Se trabaja principalmente en desarrollo rural y economía social, y no hay actividades específicas que aborden Derechos Sexuales y Reproductivos. Tan solo se abordan en transversalización del enfoque de género, y las actividades de empoderamiento económico inciden directamente sobre los derechos reproductivos y sexuales de las mujeres beneficiarias de los proyectos. Ciertamente estos derechos se deben tratar en los proyectos desarrollo local más especificamente y con mayores recursos humanos y materiales" D11

"No todos los proyectos tienen ese objetivo, aunque siempre se trata este tema, incluso con organizaciones mixtas. El cuerpo de las mujeres ha sido usado como elemento de debilitamiento del enemigo y de la comunidad, especialmente en los territorios y países donde se han dado conflictos internos de larga duración. Esto solo se corrige con el empoderamiento de las mujeres, su acompañamiento en los procesos de denuncia y solicitud de reparación" D21

En la figura 4 se puede apreciar una distribución de las organizaciones en cuanto al tipo de acciones llevadas a cabo en materia de Derechos Sexuales y Reproductivos para el periodo estudiado.

Como se puede observar, son mucho más numeras las intervenciones que se dan en el ámbito de educación frente a las que se podrían denominar como de intervención directa. Esto puede ser explicado por la propia naturaleza del mapa de ONGD en Andalucía, en el que se observan organizaciones con una clara orientación hacia la intervención son mucho menores en términos de representación numérica, a lo que hay que añadir que este tipo de intervenciones suelen precisar de un mayor nivel de especialización, de una mayor cantidad de recursos para su ejecución así como que se encuentran con mayores dificultades para ser llevadas a cabo (Coordinadora de Organizaciones para el Desarrollo España, 2019; Marrero et al., 2008).

"Porque está directamente relacionada con lo que hacemos" D3

"La organización en terreno cuenta con un Consultorio Médico, que se gestiona sobre terreno e incluye Consultas especializadas de Ginecología y Pediatría, además de Sala de Observación Materno-Infantil, Casa de Acogida y Programa de Lucha contra el Maltrato" D7

"Porque preferentemente actuamos con la infancia y mujeres en situación de vulnerabilidad" D19 
"En ese caso este tema fue identificado durante la etapa de diagnóstico" D7

"Si bien en la organización se desarrollan proyectos que abordan la temática no es una problemática priorizada. Eso no quiere decir que, en determinados contextos o momentos, la magnitud de la problemática justifique el desarrollo de intervenciones de este tipo" D14

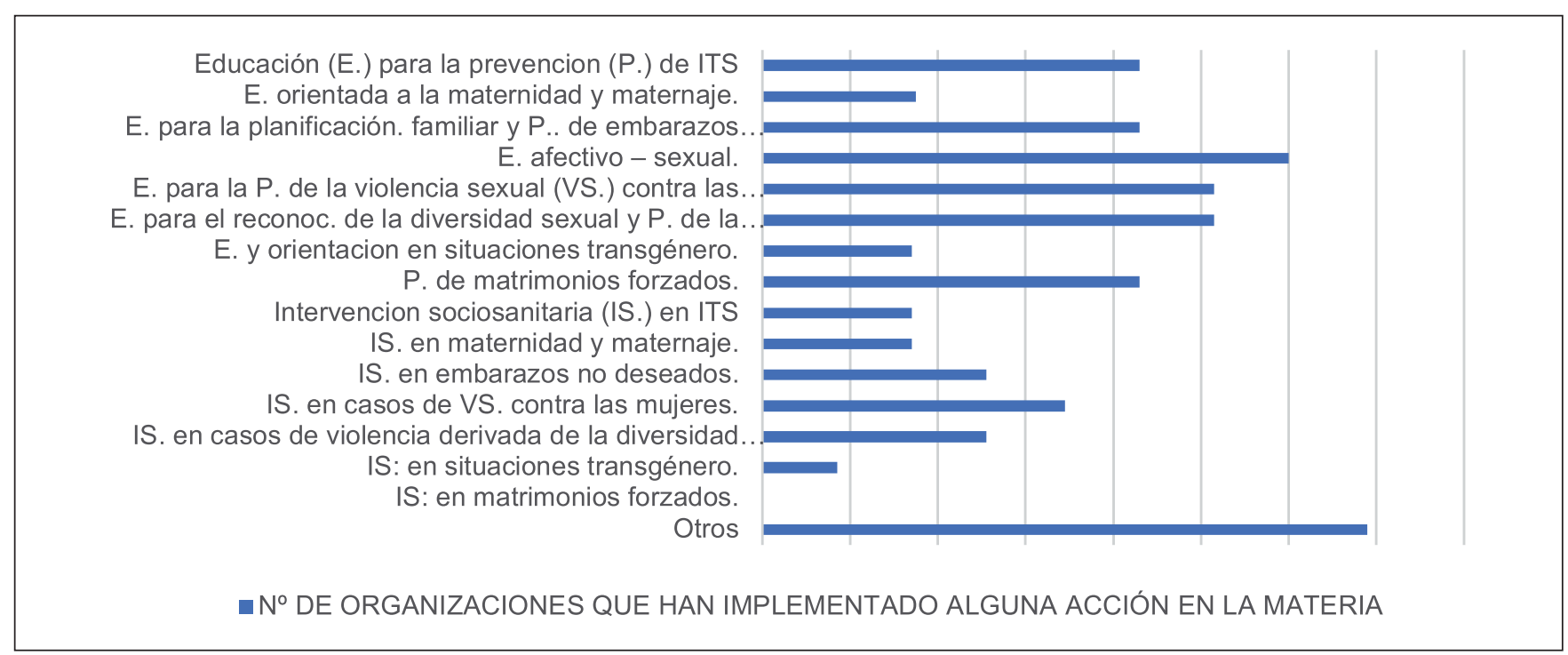

Figura 4. № de organizaciones que han implementado alguna acción en la materia. Fuente: Hernández - Ascanio (2020).

En el ámbito educativo específicamente se reconoce una mayor diversidad y heterogeneidad de acciones llevadas a cabo, pero que suelen concentrarse de forma regular en una serie de contenidos tales como la educación afectivo - sexual definida en sentido amplio, la prevención de la violencia de género y sexual, así como la promoción de las diversidades sexuales.

Esta cuestión está directamente relacionada con la consolidación de la erradicación de la violencia de género, en sus múltiples manifestaciones, como una de las prioridades marcadas por la Cuarta Ola del Feminismo (Pavón Piscitello, 2012).

Junto con lo ya expresado, las organizaciones identifican otra serie de condicionantes a la hora de decantarse por un tipo de acción u otra, teniendo mucho peso el área geográfica de actuación de las organizaciones y la configuración que se hace de los Derechos Sexuales y Reproductivos en los países en los que se llevan a cabo las acciones (Hernández-Ascanio, 2020).

En lo que respecta a cómo perciben el impacto de sus actuaciones, el $73 \%$ de las organizaciones participantes lo valoran como "alto o muy alto", a la misma vez que identifican que la mayor dificultad a superar tiene que ver con la continuidad y sostenibilidad de las acciones. Además de la cuestión económica, otro de los elementos condicionantes de la sostenibilidad de las acciones va a ser la capacidad de superar las resistencias, por cuestiones socioculturales, que se encuentran en terreno de mano de las comunidades receptoras y las organizaciones contrapartes.

"En muchos de estos países, como Guatemala, Perú, Colombia..., aún no hay un consenso en la sociedad de la gravedad de este problema y las mujeres que denuncian están sometidas a la revictimación y humillación que supone un largo proceso. No obstante, en la educación y sensibilización el impacto es mayor ya que genera un empoderamiento que les hace ser fuerte y ayudar a mujeres cercanas que padecen estas violaciones en sus entornos más cercanos" D7

"Nuestra entidad es una organización que se dedica a la educación y que está impulsada por una institución religiosa. Aunque trabaja con enfoque de género y derechos humanos, hasta este momento no había trabajado sobre el tema, el ser una organización religiosa hace que el debate y reflexión interna sea más complejo y a la vez pertinente puesto que nuestras organizaciones socias así lo demandan. Hemos comenzado un proceso de reflexión y formación interna, que irá acompañado de la ejecución de algún proyecto en el ámbito educativo en Guatemala financiado por la Junta de Andalucía, y otro en Bolivia. Proyectos que todavía no han empezado" D14 


\section{CONCLUSIONES}

Como se ha podido observar a lo largo del presente trabajo, la relación entre los Derechos Sexuales y Reproductivos sigue siendo una cuestión pendiente, en buena parte explicada por la complejidad de dicha relación, y por los múltiples elementos que entran en juego.

Afortunadamente, en sentido amplio se puede decir que el interés por el ámbito de los Derechos Sexuales y Reproductivos femeninos, ha ido creciendo a partir de iniciativas sociales comprometidas con la agenda de los movimientos de mujeres y del feminismo de las dos últimas olas (Giddens, 2000; Buss, 1998). Este interés creciente ha trascendido al ámbito de la incidencia pública a través de su reconocimiento en diferentes acuerdos internacionales (Organización de Naciones Unidas, 2015). Sin embargo, pese a esta creciente tendencia y mayor dedicación a la reflexión y a la conceptualización en este ámbito no ha tenido un suficiente reflejo en el campo de la cooperación al desarrollo, ya no sólo de la práctica situada de los agentes de cooperación, sino también de cómo este ámbito de interés configura a los propios actores. En cierta medida, nos referimos al hecho de pasar de preguntarnos ¿qué se hace en materia de Derechos Sexuales y Reproductivos en el ámbito de la cooperación internacional? a interrogarnos acerca de ¿qué significación tienen los Derechos Sexuales y Reproductivos en el ejercicio de la cooperación internacional al desarrollo? (Coordinadora de Organizaciones para el Desarrollo España, 2019).

En parte, este trabajo ha pretendido dar respuesta a esa pregunta para el caso concreto de las ONGD que operan desde Andalucía. Entre los principales hallazgos se encuentran con que los Derechos Sexuales y Reproductivos son identificados como contenidos de segundo nivel e invisibilizados dentro de ámbitos de interés de carácter mucho más amplio, como son el campo de la salud o de la promoción de género. Un número importante de organizaciones no disponen de la capacidad de situar a los Derechos Sexuales y Reproductivos de manera específica en ninguno de sus ejes de planificación estratégica, transversalizándolos, de tal forma que debilita su capacidad referenciadora. De esta manera, en las ONGD andaluzas no se observa que los Derechos Sexuales y Reproductivos "vertebren el ADN de la organización ni configuren su identidad" de una manera significativa. Esto se explica en parte por la tendencia de las ONGD andaluzas a la baja especialización.

Una segunda cuestión a tener en cuenta tiene que ver con la elevada dificultad que tienen las organizaciones para enfrentarse a la conceptualización sobre los Derechos Sexuales y Reproductivos. Esta va a ser de los principales elementos explicativos de cómo las ONDs abordan esta temática de la forma en la que lo hacen. Las organizaciones no disponen de modelos claros acerca de lo qué son, suponen y cómo deben ser abordados los Derechos Sexuales y Reproductivos en el ámbito de la cooperación al desarrollo. Sólo disponen de definiciones parciales y muy sesgadas en torno a rasgos básicos de los componentes de estos derechos. Pese a que actualmente es posible encontrar diversos modelos a partir de los cuales poder definir y proyectar las prácticas situadas de los diferentes agentes, estos, sólo hacen uso de un número restringido de enfoques entre los múltiples disponibles. Y estos, suelen coincidir con los enfoques más clásicos y asentados, especialmente los relativos a los Enfoques integrales en salud, y en menor medida, el Enfoque de derechos y el Enfoque de empoderamiento.

Sin embargo, frente a esto, los resultados muestran que las ONGD andaluzas son muy activas a la hora de implementar acciones en lo que ellos entienden que son materia de Derechos Sexuales y Reproductivos hasta tal punto que son proclives a comprometer recursos propios en la ejecución de dichas acciones. El contenido de las acciones es una fuente frecuente de tensiones tanto con las agencias habituales de financiación como con las organizaciones contraparte y poblaciones destinatarias, al existir una discrepancia en lo que respecta a la construcción teórica y los enfoques que se utilizan en el ámbito de los Derechos Sexuales y Reproductivos. Otro tipo de dificultad identificada tiene que ver con la tensión a la hora de gestionar las prácticas culturales de las comunidades y la promoción de los derechos humanos (en ocasiones identificados como ejercicios de colonialismo cultural) (Morales Hernández, 2015).

Los principales elementos que definen la práctica situada de las ONGD andaluzas en el ámbito de los Derechos Sexuales y Reproductivos son una alta convicción con la implementación de acciones, pero un déficit de claridad en su abordaje. 
Poniendo el foco en cuáles son los principales contenidos de las intervenciones llevadas a cabo por las organizaciones consultadas, se puede observar una cierta irregularidad para el quinquenio estudiado, ya que son acciones cuya sostenibilidad está muy condicionada por las cuestiones económicas y de recepción de la población destinataria que ya hemos comentado. Sin embargo, el impacto de estas percibido por las propias organizaciones es significativamente alto. En cuanto a la tipología de acciones, se identifican dos grandes bloques en torno a la educación para el desarrollo y la intervención comunitaria-asistencial. La elección entre unas acciones u otras está fuertemente condicionada por el nivel de especialización de la organización, los recursos disponibles, el área geográfica de actuación de las organizaciones y la configuración de los Derechos Sexuales y Reproductivos femeninos en dichos países.

A modo de conclusión se puede subrayar que los Derechos Sexuales y Reproductivos siguen siendo un reto de primer orden pendiente para la cooperación andaluza ya que las ONGD andaluzas se mueven entre una tensión marcada por un interés proactivo sobre los mismos y un déficit estructural de competencias para poder gestionarlos de forma eficaz.

\section{REFERENCIAS}

Arango, L. G. y Viveros, M. (2011). El género: Una categoría útil para las ciencias sociales. Bogotá: Universidad Nacional de Colombia.

Ballester Añon, R. (2015). Mujeres y culturas de la paz desde sus claves históricas. En R. Trenor Galindo (Ed.) Salud y cooperación para el desarrollo: Análisis constructivo y nuevas claves de futuro (pp. 22-33). Alicante: Universidad de Alicante.

Benhabib, S. (2010). Cultura, derechos humanos y minorías vulnerables. Una modesta propuesta. Valencia: Yale University.

Buss, D. M. (1998). Sexual Strategies Theory: Historical Origins and Current Status. The Journal of Sex Research, 35(1), 19-3. https://doi.org/10.1080/00224499809551914

Coordinadora de Organizaciones para el Desarrollo España. (2019). Memoria de actividades 2019. Madrid.

Cuesta Fernández, I. y Calabuig Tormo, C. (2010). El sistema español de cooperación interancional al desarrollo. En C. Calabuig Tormo y M. de los LI. Gómez-Torres (Eds.), La cooperación no gubernamental (pp. 91-116). Valencia: Universidad Politecnica de Valencia.

De Barbieri, T. (1999). Derechos sexuales y reproductivos: Aproximación breve a su historia y contenido. Mujer y Salud, 2.

Giddens, A. (2000). La transformación de la intimidad: Sexualidad, amor y erotismo en las sociedades modernas. Madrid: Cátedra.

Glaser, B. G. \& Strauss, A. (1967). The discovery of grounded theory: Strategies for qualitative research. Chicago: Aldine. https://doi.org/10.1097/00006199-196807000-00014

Gómez Galán, M. y Sanahuja, J. A. (1999). El sistema internacional de cooperación al desarrollo: Una aproximación a sus actores e instrumentos. Madrid: CIDEAL.

González Guerrero, S. y Pajares Sánchez, L. (2012). Guía práctica sobre derechos sexuales y reproductivos en la cooperación al desarrollo. Madrid: ACSUR Las Segovias.

González Luengo, M. R. y Gutiérrez Esteban, P. (2011). Los feminismos en el siglo XXI: Pluralidad de pensamientos. Brocar: Cuadernos de Investigación Histórica, 35, 335-351. https://doi.org/10.18172/brocar.1610

Hawkes, S. (2014). Sexual health: a post-2015 palimpsest in global health? The Lancet Global Health, 2(7), e377-e378. https://doi.org/10.1016/S2214-109x(14)70036-1

Heidari, S. (2015). El derecho a la sexualidad e integridad del cuerpo como derechos humanos. Reproductive Health Matters, 23(46), 1-6.

Hernández-Ascanio, J. (2020). Derechos sexuales y reproductivos de las mujeres como objeto de intervención en ONGD andaluzas: perspectivas, actuaciones y retos. Ciencia, Técnica y Mainstreaming social, 4, 13-28. https://doi.org/https://doi.org/10.4995/citecma.2020.12204

Jelin, E. (1993). ¿Ante, de, en, y? Mujeres, Derechos Humanos. Lima: Red Entre Mujeres.

Khosla, R., Say, L. \& Temmerman, M. (2015). Sexual health, human rights, and law. The Lancet, 386(9995), 725-726. https:// doi.org/10.1016/S0140-6736(15)61449-0

Lagarde y de los Ríos, M. (2005). Para mis Socias de la Vida. Madrid: Horas y Horas. 
Lozano, I. (1999). La salud reproductiva en el marco de la cooperación al desarrollo. Afers Internacionals, 51-52, 189-204.

Marrero, B., O’Kelly, M., Solsona, M. y Zumarán, A. (2008). Políticas europeas de cooperación al desarrollo en materia de salud sexual y reproductiva. Madrid: Fundación Carolina.

Morales Hernadez, L. A. (2015). Violencia cultural, colonialismo y reetnización; el sentido de las prácticas en salud desde el punto de vista indígena. Revista de la Facultad de Medicina, 63(4), 699-706. https://doi.org/10.15446/revfacmed. v63.n4.50049

O’Brien, B. C., Harris, I. B., Beckman, T. J., Reed, D. A. \& Cook, D. A. (2014). Standards for Reporting Qualitative Research. Academic Medicine, 89(9), 1245-1251. https://doi.org/10.1097/ACM.0000000000000388

OEA. (1994). Convencion interamericana para prevenir, sancionar y erradicar la violencia contra la mujer «Convencion de Belem do Para».

Olmo Gascón, A. M. (2018). La instrumentalización de la sexualidad y reproducción femenina en el sistema productivo y comercial global. En Diversidad sexual y libertad reproductiva de las mujeres en la cultura de la producción y el consumo (pp. 175-192). Granada: Comares.

ONU (1979). Convención sobre la eliminación de todas las formas de discriminación contra la mujer.

ONU (1994). Conferencia Internacional sobre la Población y el Desarrollo en el Cairo.

ONU (1995). Report of the Fourth World Conference on Women.

ONU (2000). Declaración del Milenio de Naciones Unidas. Nueva York.

Organización de Naciones Unidad Mujeres (2016). No Titl. Recuperado de https://www.unwomen.org/es/news/in-focus/ women-and-the-sdgs

Organización de Naciones Unidas (2015). Agenda 2030 para el Desarrollo Sostenible.

Pavón Piscitello, D. (2012). Igualdad de género y cooperación internacional para el desarrollo: Género y desarrollo. En M. S. Ojeda (Ed.), Género y mujer desde una perspectiva multidisciplinar (pp. 271-278). Madrid: Fundamentos.

Pérez de Armiño, K. (2000). Diccionario de acción humanitaria y cooperación al desarrollo. Bilbao: Icaria - Hegoa.

Prado Lallande, J. P. (2010). La condicionalidad de la ayuda y el enfoque de derechos humanos: propuestas prácticas para la cooperación española. Madrid: CeALCI- Fundación Carolina.

Shallat, L. (1993). Derechos de la vida. Mujer y Salud, 3.

Yamin, A. E. (2019). Silencing the drama - Do the SDG indicators expose the injustices that limit women's sexual and reproductive lives? Recuperado de https://www.openglobalrights.org/silencing-the-drama-do-the-SDG-indicators-expose-the-injustices-that-limit-womens-sexual-and-reproductive-lives/?lang=English 


\section{ANEXO}

\section{CUESTIONARIO “DERECHOS SEXUALES Y REPRODUCTIVOS DE LAS MUJERES EN LAS ONGD ANDALUZAS”}

Estimados/as amigos/as, desde el Área de Sociología de la Universidad de Córdoba estamos desarrollando un trabajo de investigación para el cuál solicitamos su importante ayuda. El objetivo de dicho trabajo es conocer un poco más en profundidad cuál es el papel de las ONGD andaluzas con respecto a la promoción y defensa de los Derechos Sexuales y Reproductivos en el ámbito de la cooperación internacional y de la educación para el desarrollo. Es por ello por lo que nos ponemos en contacto con ustedes solicitándoles que cumplimenten el siguiente cuestionario que no les llevará más de unos breves minutos.

Dicho cuestionario es totalmente anónimo y los resultados que en él se recogen se hará de forma totalmente desagregada a fin de mantener la privacidad de los datos en él reflejados. El cuestionario formula preguntas relativas a la concreción de los Derechos Sexuales y Reproductivos en la identidad, misión y valores de su organización, del tipo de actuaciones que llevan a cabo y del impacto de estas. Consideramos que su ayuda es de suma importancia, por lo que agradecemos su colaboración en este trabajo. Para cualquier tipo de aclaración en relación con el presente cuestionario o la investigación en la que se inserta se podrá hacer mediante contacto con el correo jhascanio@uco.es

- Nombre de la Organización

- Email address.

1. Indique el ámbito preferencial de actuación de su organización:

- Promoción comunitaria

- Educación para el Desarrollo

- Ayuda humanitaria

- Promoción de la salud

- Promoción de la Igualdad

- Mixto

2. ¿Podría definir brevemente lo que en su organización se consideran "derechos sexuales" y "derechos reproductivos"?

3. ¿Considera que "derechos sexuales" y "derechos reproductivos" son elementos indisociables o por lo contrario son derechos autónomos uno respecto a los otros?

- Derechos indisociables

- Derechos autónomos

4. ¿Podría justificar brevemente su respuesta anterior?

5. ¿Podría indicar cuál es el posicionamiento político de su organización con respecto a los Derechos Sexuales y Reproductivos?

- Enfoque de derechos

- Enfoque de salud

- Enfoque de ciudadanía y laicismo.

- Enfoque de autonomía y empoderamiento.

- Enfoque intercultural.

- $\quad$ No existe ningún enfoque explicitado.

6. ¿En qué ejes de Planificación Estratégica de su organización se mencionan los Derechos Sexuales y Reproductivos?

- Salud

- Género

- Educación

- Democracia y sociedad civil

- Gobernanza

- Otro

- Ninguno 
7. ¿Ha desarrollado alguna actividad en el ámbito de "derechos sexuales" o "derechos reproductivos" en los últimos 5 años?

- Sí

- No

8. ¿Ese proyecto ha sido financiado por alguna entidad pública?

- Sí

- No

9. ¿Podría indicar cuál?

10. ¿Podría indicar con qué porcentaje económico con respecto al total?

11. ¿Considera que la financiación de proyectos en el ámbito de los Derechos Sexuales y Reproductivos se encuentra condicionado por aspectos ideológicos / políticos de las entidades financiadoras? (0 se corresponde con "en absoluto" y 5 con "totalmente).

12. ¿Considera que la actuación que está llevando a cabo tiene una asignación de recursos suficiente acorde a la importancia que reconoce?

- Sí

- No

13. En el caso de haber contestado que no a la anterior pregunta, ¿puede indicarnos la causa de esta financiación insuficiente?

14. ¿Podría indicar el país o países en los que se han desarrollado dichos programas?

15. ¿De qué manera están contemplados los Derechos Sexuales y Reproductivos en la constitución y legislación del país/región donde se lleva a cabo la actuación?

- Los Derechos Sexuales y Reproductivos están totalmente reconocidos

- Los derechos sexuales están parcialmente reconocidos

- Los derechos sexuales están totalmente reconocidos

- Los derechos reproductivos están parcialmente reconocidos

- Los derechos reproductivos están totalmente reconocidos

- Los Derechos Sexuales y Reproductivos están totalmente excluidos de la legislación.

- Desconozco el nivel de reconocimiento de dichos derechos.

16. ¿Cuál es la aplicación práctica del reconocimiento legal de los Derechos Sexuales y Reproductivos en esos países?

- Los Derechos Sexuales y Reproductivos están totalmente reconocidos en la práctica.

- Los derechos sexuales están parcialmente reconocidos en la práctica.

- Los derechos sexuales están totalmente reconocidos en la práctica.

- Los derechos reproductivos están parcialmente reconocidos en la práctica.

- Los derechos reproductivos están totalmente reconocidos en la práctica.

- Los Derechos Sexuales y Reproductivos están totalmente excluidos de la legislación en la práctica.

- Desconozco el nivel de reconocimiento de dichos derechos en la práctica.

17. ¿Podría indicar si los proyectos llevados a cabo han incluido algunas de las siguientes acciones?

- Educación para la prevención de Infecciones de Transmisión Sexual.

- Educación orientada a la maternidad y crianza.

- Educación orientada a planificación familiar y prevención de embarazos no deseados.

- Educación afectivo - sexual.

- Educación para la prevención de la violencia sexual contra las mujeres.

- Educación para el reconocimiento de la diversidad sexual y prevención de la violencia asociada a la misma.

- Educación y orientación en situaciones transgénero / transexual.

- Prevención de matrimonios forzados.

- Intervención socio - sanitaria en infecciones de transmisión sexual.

- Intervención socio - sanitaria en maternidad y crianza.

- Intervención socio - sanitaria en embarazos no deseados 
- Intervención socio - sanitaria en casos de violencia sexual contra las mujeres.

- Intervención socio - sanitaria en casos de violencia derivada de la diversidad sexual.

- Intervención socio - sanitaria en situaciones transgénero / transexual.

- Intervención socio - sanitaria en matrimonios forzados.

18. ¿Los sujetos preferenciales de estos proyectos han sido?

- Mujeres

- Hombres

- Indistinto género

19. En caso en que haya contestado en la anterior pregunta "educación para la prevención de la violencia sexual contra las mujeres o en situaciones transgénero / transexual" o "intervención socio - sanitaria en casos de violencia sexual contra las mujeres o en situaciones transgénero / transexual", ¿podría concretar cuál ha sido la actuación?

- Violación dentro o fuera del ámbito de la pareja.

- Violación en contextos de conflictos armados y relacionados a prácticas de guerra.

- Trata de seres humanos con fines sexuales.

- Prostitución infantil y adolescente.

- Abuso sexual infantil y adolescente.

- Mutilación genital.

- Otros

20. Los sujetos preferenciales de estos proyectos han sido:

- Mujeres

- Hombres

- Indistinto Género

21. ¿Podría justificar brevemente el por qué ha optado su organización por desarrollar esos proyectos frente a otros de diferente temática?

22. ¿En la definición del problema y de las actuaciones han participado sujetos afectados por la problemática?

$-\mathrm{Si}$

- No

23. En caso de contestar afirmativamente a la anterior pregunta, indique cuáles han sido los mecanismos para facilitar dicha participación.

24. En caso de contestar negativamente a la anterior pregunta, indique cuáles han sido los motivos.

25. En relación con el impacto de las actuaciones que está llevando a cabo, considera que el mismo es

- Muy bajo

- Bajo

- Medio

- Alto

- Muy Alto

26. En caso de que haya contestado "bajo" o "muy bajo", ¿puede indicarnos cuál o cuáles considera que son los principales motivos para ello? 